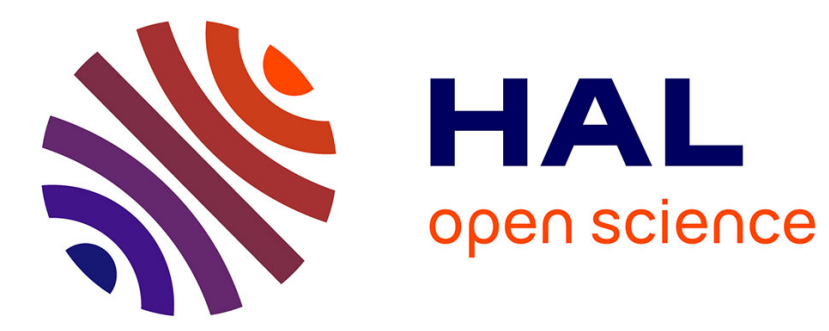

\title{
Les enfants des marchands de Kaniš
}

Cécile Michel

\section{- To cite this version:}

Cécile Michel. Les enfants des marchands de Kaniš. KTÈMA Civilisations de l'Orient, de la Grèce et de Rome antiques, 1997, 22, pp.91-108. halshs-00821268

\section{HAL Id: halshs-00821268 https://shs.hal.science/halshs-00821268}

Submitted on 8 May 2013

HAL is a multi-disciplinary open access archive for the deposit and dissemination of scientific research documents, whether they are published or not. The documents may come from teaching and research institutions in France or abroad, or from public or private research centers.
L'archive ouverte pluridisciplinaire HAL, est destinée au dépôt et à la diffusion de documents scientifiques de niveau recherche, publiés ou non, émanant des établissements d'enseignement et de recherche français ou étrangers, des laboratoires publics ou privés. 
Les enfants des marchands de Kaniš

RÉsumé. - Les marchands assyriens installés en Asie Mineure au début du second millénaire avant J.-C. ont laissé de très nombreuses archives privées qui documentent divers aspects de leur vie quotidienne et de celle de leur famille, souvent déchirée entre Ašsur et Kaniš. Les enfants de ces marchands, quoique peu attestés dans les textes, y interviennent toutefois par l'intermédiaire d'un vocabulaire riche et varié. parents et autres membres de la famille. Quelques allusions aux enfants permettent d'envisager leur statut juridique, esclaves et enfants libres, et leur droit en matière d'héritage.

ABSTRACT. - At the beginning of the second millenium B.C., the Assyrian merchants who settled in Asia Minor left many private archives. They document several aspects of their family daily life frequently torn between Assur and Kanis. The mentions of merchants children, although rare in the texts, occur however through a rich and miscellaneous vocabulary. They are mentioned by the people in charge of their care and education, parents, grand-parents and other members of the family. A few references about children allow us to study their legal statute, slaves and free children, and their inheritance rights.

En deçà d'un certain âge, les enfants des marchands assyriens exerçant en Cappadoce ne participent pas au commerce de leurs parents. Par conséquent, les archives privées des marchands de Kaniš restent plutôt silencieuses sur ces enfants en bas âge et sur leur éducation. Néanmoins, les mentions d'enfants existent non seulement dans la documentation juridique, mais aussi et surtout dans la correspondance privée des couples ou de plusieurs membres d'une même famille.

L'analyse du vocabulaire relatif à l'enfant dans les tablettes paléo-assyriennes permet d'aborder cet âge de la vie sous différents aspects. Les références aux enfants dans les lettres émanent souvent des individus en charge de leur entretien et de leur éducation. Plusieurs membres de la famille, en dehors des parents, peuvent être amenés à élever les enfants; on relève les principales préoccupations de ces derniers vis-à-vis des enfants sous leur responsabilité. Enfin, le statut des enfants ou encore leurs droits peuvent être abordés à partir de rares textes juridiques ou encore de brèves allusions dans les missives.

\section{Vocabulaire afFÉr Ent à L'EnFant}

Le vocabulaire relatif à l'enfance, bien que riche et varié, ne comprend, dans certains cas, que de rares occurrences. Les termes recensés peuvent être ordonnés selon les thèmes suivants : la naissance, les enfants asexués, les filles et les garçons et les verbes «être petit» et "grandir».

\subsection{La naissance}

La naissance d'un enfant est indiquée par le verbe walädum qui signifie «donner naissance à» ou encore «accoucher». Ce terme, rare en paléo-assyrien, n'intervient qu'en deux circonstances 
bien particulières : dans les textes juridiques $\left({ }^{(}\right)$, ou encore lors de la mention d'une date faisant référence à un événement princier. En ce qui concerne la documentation juridique, employé lans un aontrat d'adoption (TCL 1240), ce verbe permet d'envisager la naissance d'un héritier direct du père adoptif. Ce texte concerne un couple d'Anatoliens qui, n'ayant sans doute pas pu donner naissance à un enfant, en adopte un. Différentes clauses règlent le statut de l'enfant adopté dans sa nouvelle famille, ainsi que ses droits en matière d'héritage. A cette occasion la naissance éventuelle d'un enfant mâle qui pourrait porter ombrage à l'adopté est envisagée ( ${ }^{2}$ ) «Si (une femme) de Habatali donne naissance à un garçon pour lui».

En second lieu, la naissance d'un enfant princier peut servir à dater une reconnaissance En Dame-de-la-Maison a accouchér. La Dame-de-la-Maison représente très vraisemblablement la souveraine anatolienne, la Maison correspondant au palais princier.

\subsection{Les enfants en général}

Le vocabulaire relatif aux enfants est représenté par deux catégories de mots. Le premier ensemble ne comprend que des termes asexués et regroupe essentiellement les enfants les plus jeunes.

\subsection{1. lipum}

Le mot thpum n'est jusqu'à présent attesté qu'une seule fois dans la documentation paléoassyrienne. Intervenant dans un contrat de mariage, il sert à désigner les futurs enfants des nouveaux époux $\left({ }^{4}\right):$ «Si dans les deux ans elle (l'épouse) ne lui a pas procuré de descendance...»; il s'agirait donc d'un terme générique pour désigner les enfants non encore conçus, mais à venir.

\subsection{2. ša/errum}

Le mot šerrum, souvent vocalisé en «a» en paléo-assyrien, peut également, mais de façon exceptionnelle, désigner la descendance à venir ( $\left.{ }^{(}\right)$: «Si IStar-lamassī n'a pas d'enfant dans les 3 ans...». Mais il intervient aussi en opposition à tipum pour désigner un enfant né $(6)$ : «Et

(1) Le verbe walädum n'intervient pas dans le texte $T C 3214 \mathrm{a}, 7$, contrairement à ce qu'avait transcrit K. BALKAN, Letter of King Anum-Hirbi of Mama to King Warshama of Kaniš, Ankara 1957, p. 46. En effet, selon V. Donbaz, qui a collationné la tablette, le verbe est à lire e-ha-az, «Some Remarkable Contracts of I-B Period Kültepe Tablets», dans K. Emre, B. Hrouda, M. Mellink et N. Ozgéç (éds.), Anatolia and the Ancient Near East, Studies in Honor of Tahsin Özgǘ, Ankara 1989, p. 79.

(2) TCL 1 240, 12-13: šu-ma sa Ha-ba-ta-li, sú-ha-ar-šu ú-lá-ad. Cette expression est reprise à la ligne 23 : šuma sú-ha-ra-am ú-lá-ad, «Si elle donne naissance à un garçon». Le sujet du verbe walädum, dans cette seconde occurrence peut désigner la femme du couple, ou encore une épouse secondaire, que le mari aurait prise afin d'avoir un descendant. (3) $\mathrm{Kt} \mathrm{a} / \mathbf{K}$ 851, 7-9: 3 gin kù.gi, i-nu-mì be-lá-at, éce-tim tù-ull[du], K. BALKAN, «The Conception of Trinity in the Tablets of Kültepen, dans H. OTten, E. AKurgal, H. ErTEM et A. SUEL (éds.), Hittite and other Anatolian and Near Eastern Studies in Honor of Sedat Alp, Ankara 1992, p. 20-21. L'editeur de ce texte relève l'existence egalement de noms propres formés sur le verbe wal̄ondum, tel Waldill, $66,3,6=E L 220)$

(4) ICK $13,7-9$ : ̌̌u-ma : a-dí, mu 2-sè li-pè-e, lá ta-ar-ti-sí-su-um.

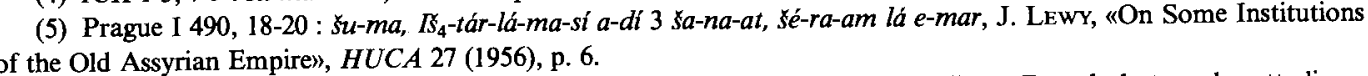
of the Old Assyrian Empiren, HUCA 27 (1956), p. 6

政, ff. H. HIRSCH, «Eine Kleinigkeit zur Heiratsurkunde ICK 1, 3n, OrNS 35 (1966), p. 280. plus tard, après lui avoir procuré un enfant de cette manière...». Alors que dans le premier cas on envisage l'existence d'une éventuelle descendance, ici, l'auteur de ce contrat de mariage évoque l'hypothèse où l'enfant du couple est déjà né.

De façon plus générale, le mot $\Varangle a / e r r u m$, le plus souvent employé au pluriel, désigne les enfants en bas âge d'une famille dans leur ensemble. Une traduction de ce mot par "progéniture" convient parfaitement à son utilisation dans les clauses de garantie des contrats de prêt. Le ša/errum se situe alors à côté de la femme du débiteur (\urcorner$)$ : «(Relativement au versement de) l'argent ils sont solidairement responsables de même que leur femme et leur progéniture». Parfois, il apparaît également au début d'une reconnaissance de dette, et constitue alors l'un des membres du groupe débiteur $\left.{ }^{8}\right)$ : «Enišru et Tamura ont (en créance) 2 mines 12 sicles d'argent sur Amur-Ał̌sur, fils d'Alu, Zazhuna, sa femme, sa progéniture et sa maison». Dans ces différents documents juridiques, les enfants du débiteur servent donc de garantie au créancier, comme cela peut être explicitement précisé $\left({ }^{9}\right)$ : «(Jusqu'à ce que le prêt soit remboursé), ses esclaves, sa maison, ses enfants et ses champs sont ceux d'Ikūnum".

En dehors d'un contexte juridique, šerrum est utilisé par les marchands dans un sens toujours très général pour désigner les petits enfants d'une famille dans leur ensemble. Le plus souvent, il apparaît pour désigner les enfants des collègues ou des serviteurs :

$$
\begin{aligned}
& \text { «Kiama et ses enfants» }\left({ }^{10}\right) \text {, } \\
& \text { "tes enfants et ta femme» }\left({ }^{11}\right) \text {, } \\
& \text { «tes enfants» (12). }
\end{aligned}
$$

Enfin, ce mot est employé dans une inscription votive rédigée à la troisième personne du singulier et commandée par Hattîtum, femme d'un certain Enna-Da[gan], dans le but de protéger sa famille $\left({ }^{13)}\right.$ : «(Pour la vie de son mari, pour sa vie et) pour la vie de ses enfants». Les différentes inscriptions votives assyriennes munies de la formule ana balāt ne mentionnent pas toujours les enfants. Lorsque ceux-ci apparaissent, ils figurent systématiquement en dernière position, et il s’agit uniquement du fils ou des fils de l'intéressé ( ${ }^{14}$ ). Dans le cas présent, łerrum dénote sans doute tous les enfants, filles ou garçons, déjà nés de Hattītum, ainsi que ceux à venir, et peut par conséquent être traduit par «descendance».

\subsection{3. şuhrum}

Le mot le plus fréquemment utilisé par les marchands de Kaniš relativement à leurs enfants ou à ceux de leurs collègues et amis est le mot suhrum. Singulier collectif, suhrum est le

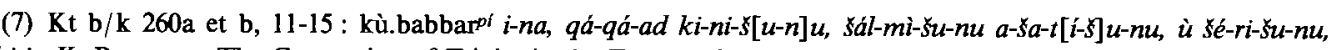
ra-ki-is, K. Balkan, «The Conception of Trinity in the Tablets of Kültepe», dans H. Otren, E. Akurgat, H. ERTEM et A. SUEL (éds.), Hittite and other Anatolian and Near Eastern Studies in Honour of Sedat Alp, Ankara 1992, p. 41-44.

(8) TC 3 237, 1-6: 2 ma-na 12 gín kù-babbar, i-sé-er A-mur-a-sur dumu A-lu, Za-az-hu-na a-sit-ti-su, sa-ri-š

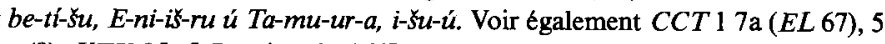

(9) KTK 95, 57 ; géme ir é [?], sa-ru-su ú eq-Íá-tum], sa I-ku-num, cf. K. R. VEenHOF, BiOr 27 (1970), p. 36 369. Voir également le texte $K T K$ 96, 1516 : é-sú ú a-sa-sú, ú łé-ru-šu a-da-gal, «Je possède sa maison et sa femm ses enfantsw.

(10) CCT 37a, 32: Ki-a-ma ú séri-š.

(12) KTS 223, $17:\langle e-r i-k \dot{a}$.

(13) Cette inscription votive retrouvée dans le temple d'Istar à Ašsur, VA Ass 4286, a été publiée par L. JАKOB-

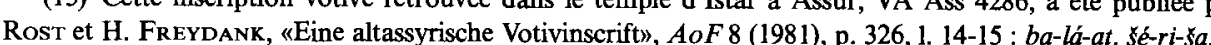

(14) K. DELLER, «Zum ana bälät formular Einiger Assyrischer Votivinschriften», OrAnt 22 (1983), p. 13-24. 
terme générique pour désigner les enfants. Il intervient dans quelques contextes caractéristiques des principales préoccupations des adultes envers les plus jeunes $\left({ }^{15}\right)$.

Les enfants-suhrum, entre sevrage et adolescence, constituent l'un des éléments de la maisonnée ${ }^{16}$ ). Le couple de marchands tient compte de leur existence dans la gestion de son budget : il faut les habiller et les nourrir. Suhrum englobe les garçons et les filles d'une même famille $\left({ }^{17}\right)$, le marchand ayant recours à d'autres termes lorsqu'il ressent le besoin de préciser le sexe de l'enfant $\left({ }^{18}\right)$

Le vocabulaire retenu dans cette catégorie concerne donc les enfants en général, depuis la descendance à venir jusqu'au jeune enfant pas encore pubère.

1.3. Filles et garçons

Le second vocabulaire recensé réunit des mots qui précisent le sexe de l'enfant: fille ou garçon.

\subsection{1. şahhartum et şuhārtum şahhertum}

Le terme sahhartum désigne «la petite fille»; son équivalent masculin n’a pas encore été relevé dans la documentation paléo-assyrienne disponible. Plus jeune que la suhārtum, la petite fille-sahhartum constitue un élément de la progéniture-šerrum du marchand. En effet, une lettre offre la mention juxtaposée de ces deux derniers termes, le premier étant vraisemblablement une sous-partie du second $\left({ }^{19}\right)$.

Il semble néanmoins que l'emploi du terme şahhartum pour désigner les jeunes enfants de sexe féminin ne soit pas d'usage courant dans la documentation paleo-assyrienne, attendu que l'on trouve une autre expression, également peu répandue qui renvoie à cette même catégorie. De fait, l'adjonction de l'adjectif şhhertum au mot şuhārtum «la jeune fille», peut être traduit par "la toute-petite fille» $\left({ }^{20}\right)$.

\subsection{2. șuhārum et şuhārtum}

Les termes suhārum et suhārtum relèvent d'un usage multiple dans les archives des marchands de Kaniš. Ils renvoient parfois aux enfants, mais le plus souvent ils servent à désigner la domesticité et doivent alors être traduits par «serviteur» et «servante».

(15) Les marchands s'schangent des nouvelles des enfants et du reste de la famille (BIN 491, 35, BIN 617 , 24, CCT $238,33, C C T 428 \mathrm{~b}$, 27). Ils doivent assurer la subsistance des enfants (BIN $411,18, B I N 422,23$, $B I N 467,12, B I N 6124,15, B I N 6183,9,15, B I N 6197,14, K T K 19,35, K T S 19$ a, 19), et les vêtir $(A K T$ 2 52, 10, BIN 4 68, 11, BIN 6 84, 31, BIN 6 184, 8, BIN 6 249, 9, CCT 3 20, 21, Kt 75/k 78, 2, 29, KTS $31,3)$. Ils s'occupent de leur progéniture et la protègent (BIN $4219,8, I C K 165,17$, Kt 93/k 331, 18, TC 393 , 12, 16, TPAK 1 22, 24, VS 2616 , CG.). Enfin, les enfants apparaissent en diverses occasions (TC 276 (1)

Plusieurs lettres, Ban Ta maison et tes enfants se portent bien»; voir également $B I N 491,35$

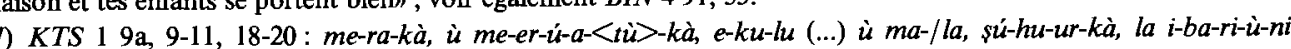
"Ton fils et tes filles ont (tout) mange (...), aucun de tes enfants ne doit mourir de faim !"

(18) II utilise alors les mots mārum «fils" et mārtum (sille», ou encore suhārum "garçon» et suhārtum «fille».

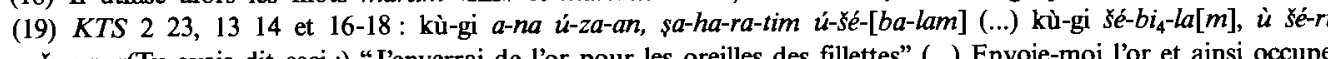
$k a ̀, a-\zeta ̌-u r$, «(Tu avais dit ceci :) "J'enverrai de l'or pour les oreilles des fillettes" (...) Envoie-moi lor et ainsi occupetoi de tes enfants", cf. W. FARBer, Aula Or 8 [1990], p. 144.

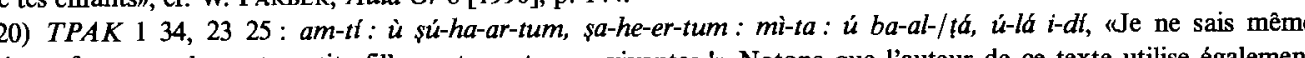

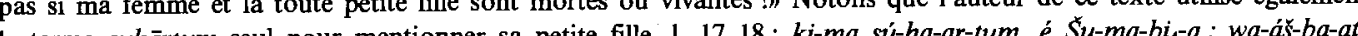
ni, «(II m’a dit) que la (petite) fille resside (dans) la maison de Sumī-abìya.
Les emplois du féminin şuhārtum peuvent être définis de façon plus précise que ceux du masculin suhārum. Selon les contextes, le mot suhārtum peut être successivement interprété sous les traductions suivantes: «servante», «jeune fille (en âge d’être mariée)», «fille», «petite fille», et il peut également correspondre à un nom propre, Suhartum (21). Ce terme est exceptionnellement attesté comme l'un des enfants-šerrum d'un individu et correspond alors à une fillette $\left({ }^{22}\right)$. D'autres références renvoient sans doute aussi à une petite fille, objet d'inquietude des parents. Un marchand désigne ainsi sa propre fillette dont petile objet nouvelle; il emploie alors successivement le terme suhārtum et l'expression suhärtum sahhertum dans le même sens (ci-dessus note 20). Le plus souvent, lorsqu'il ne s'agit pas d'une domestique, le mot şuhărtum renvoie à une fille en âge d'être vouée à une divinité ou encore d'être mariée. C'est ainsi que Lamassī, dans ses nombreuses lettres envoyées à son époux, Pūsu-kēn, nomme sa fille qu'elle désire vouer à une divinité ${ }^{(23)}$. De même, suhāartum est fréquemment nomme sa fille qu'elle désire vouer à une divinité ${ }^{(23)}$. De même, șuhārtum est fréquemment employé lorsqu'il s'agit du mariage d'une jeune fille (24).

De même que son équivalent féminin, şuhārum se traduit le plus souvent par userviteun (25), et plus rarement par «garçon» ou «jeune garçon». De fait, il apparaît occasionnellement comme l'un des enfants-şuhrum, et sert alors à préciser le sexe de l'enfant mentionné (26). En outre, le mot şuhārum intervient dans des expressions parallèles à celles qui sont recensées pour le terme suhrum ${ }^{(27)}$; ce dernier usage confirme que les deux termes sont parfois synonymes. Plus qu'un enfant, le şuhārum est avant tout un garçon et ce mot est éventuellement utilisé pour envisager la naissance d'un enfant mâle ${ }^{(28)}$. Il sert ensuite à désigner le jeune garçon, sans doute déjà sevré, mais qui demeure à élever ${ }^{29}$ ).

En bref, şuhārtum et şuhārum correspondent, dans les exemples cités ci-dessus, respectivement à la fille et au garçon de la petite enfance à l'adolescence.

\subsection{3. mārum et mārtum}

Contrairement au vocabulaire déjà analysé et qui correspond aux différentes classes d'âge de l'enfance, les mots mārum «fils» et mārtum «fille» relèvent du lexique relatif aux liens de

(21) TC 167 et le commentaire de M. ICHISAR, «Un contrat de mariage et la question du lévirat à l'époque cappadocienne", $R A 76$ (1982), p. 168-173.

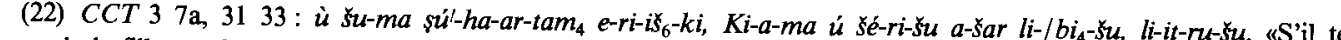
demande la fillette, alors qu'il emmène Kiama et ses enfants là où il le désiren. Kiama étant un nom propre masculin il est probable que la şuhārtum mentionnée 1. 31 corresponde à l'une de ses enfants. La suhārtum pourrait peutctre aussi constituer un sous-ensemble des enfants-suhrum dans le texte $B I N 468,12$, si l'on considère qu'li ne s'a pas là d'une servante, 9-13: 3 gín kù-babbar, ku-nu-ki-a ù 1 túg kam-sú-um : sa súüü $[h]-r i-/ i m$, a-na sú-ho-ar-tim Puzur $_{2}-a-s u r$ : na-as-a-ki-im, «Puzur-Ašsur t'apporte 3 sicles d'argent à mon sceau et une étoffe kamsum (pour vetement) d'enfant pour la fillettem.

(23) $B I N 49,20-23, C C T 320,38-40$ et $R A 59$ (1965), p. 159, 311.

(24) 276 , 27 ou encore Kt $84 / \mathrm{k} 281$ publiée par S. Bayram et S. Çeçen, «6 Neue (25) Tel est le cas du Schid

(2S) Telest le cas du texte $T C 1 \mathrm{H}$, où ce mot est associè aux propriétés mobilières et immobilières d'un individu 82 (1992). Notons que J. HENGSTL, "Soll und Haben" in einer altassyrischen familienrechtlichen Urkunden, $Z A$

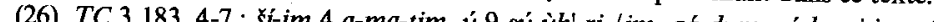

(26) un jeu $183,4-7:$ it-im 4 a-ma-tim, u 9 súu-üh-ri-/im, qd́-dum, sú-ha-ri-im, «(...) prix de 4 servantes et 9 enfants,

(27) VS 269, 23-24: bé-et-kà, ù şú-ha-ru-kà, sál-mu, «Ta maison et tes enfants se portent bien».

(29) BIN 4 129, 4, CCT2 36a, 23 ou HUCA sup. 3 n. 31 (L29-590), 11 et 21. 
parenté et, de ce fait, ne devraient pas être intégrés à cette étude. Néanmoins, quelques rares emplois de ces deux mots ressemblent aux précédents et doivent donc être pris en compte. pestrer un individu à l'aide de son patronyme. Ils peuvent toutefois être utilises à la place des enfantssuhrum afin d'en préciser le sexe : «Ton fils et tes filles» est repris un peu plus loin par «tes enfants" ( ${ }^{(30}$ ). Par ailleurs, ces mots apparaissent dans des contextes identiques à ceux dans lesquels on trouve «les enfants», filles ou garçons. Il faut de même les nourrir et les élever ( $\left.{ }^{31}\right)$, et ils figurent aussi comme garanties parmi les possessions du débiteur ${ }^{32}$ ).

Cette seconde catégorie de termes relatifs à l'enfance renferme donc approximativement préCette seconde categorie de termes relatirs à lenfance renteme plus jeunes enfants.

\section{4. être petit et grandir}

Deux verbes enfin, associés à l'enfance en général, ont été relevés. L'un projette l'enfant dans le futur, tandis que l'autre permet à l'adulte de rappeler des événements qui eurent lieu dans sa jeunesse.

\subsection{1. rabûm "grandir»}

Le verbe rabûm «grandiny, utilisé au système I, insiste sur la croissance de l'enfant ; il intervient le plus souvent pour indiquer qu'un enfant, généralement du sexe féminin, a atteint l'âge de quitter sa famille. Conjugué au parfait, rabûm s'applique ainsi aux filles en âge d'être vouées à une divinité ( ${ }^{(3)}$ ), ou encore d'être mariées ( ${ }^{34}$ ). Par ailleurs, l'usage de rabûm permet de préciser le lieu dans lequel un enfant donné doit grandir ${ }^{35}$ ) ; ce dernier emploi renvoie à l'utilisation de ce verbe au factitif. De fait, au système II, rabûm signifie «éleven», et peut concerner le lieu où l'enfant est élevé ( ${ }^{30}$ ), ou plus simplement le fait même d'élever un enfant ( ${ }^{(37)}$.

Enfin, un autre emploi de la racine RB' doit être mis en relation avec le coût de l'éducation de l'enfant. Il s'agit du terme tarbìtum, qui correspond en paléo-assyrien à «un montant d'argent pour élever un enfant», et équivaut en quelque sorte à notre «pension alimentaire» $\left({ }^{38}\right)$. Cette

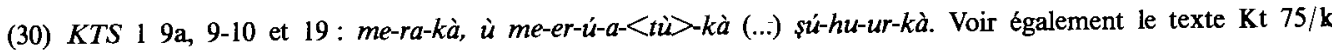

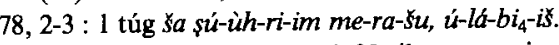

(31) Dans le texte $I C K$ 132, il est question d'argent pour élever une fille, tarbìtum, de même que dans $H U C A$ sup. 3 n. 31 (L29-590), où le mot tarbīum est associé à suhärum. CCT 36 b, fils et filles sont élevés par le grandpère (ci-dessous). Enfin, de même que les autres mots servant à désigner les enfants, mārum et märtum apparaissen , direct du verbe nourrir (SKL III) : KTS 18 b, 3-9 ou KUG 27, 52.

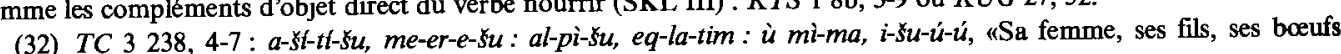
tout ce qu'il posseden.

(33) Voir le dossier relatif à la fille de Pūsu-kēn et Lamassī : CCT 320,39 (ir-té-bi) et BIN 49,21 (ir-té-bít) (34) $\mathrm{Kt} \mathrm{i} / \mathrm{k}$ 120, 4-5, publiée par K. BALKAN, «Betrothal of Girls during Childhood in Ancient Assyria and Anatolia»,

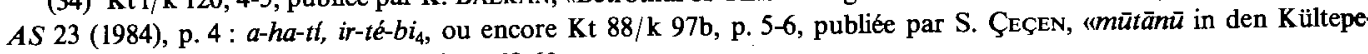
Texten», $\operatorname{ArAn} 1$ (1995), p. 56-57, copie p. 68-69.

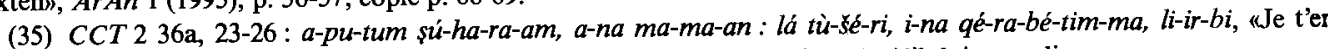
. (36) BIN $4129,3-4: a-s a r$, şu-ha-ra-am úu-ra-bu-ú-ni, «Là où ils elèveront le garçon».

t j'ai élevé tes filles». sicles d'argent) pour élever le petity. somme d'argent comprend pour l'essentiel le coût de la nourriture de l'enfant, et le terme tarbītum peut être alors remplacé par le mot ukultum «nourriture» ( ${ }^{39}$ ).

1.4.2. şahārum «être petit»

A l'inverse de rabûm, qui envisage l'avenir de l'enfant, le verbe şahārum, au permansif, permet à l'adulte de rappeler son enfance, des faits qui se sont alors produits $\left({ }^{(40}\right)$, ou des relations tissées dès cette période avec son entourage ${ }^{41}$ ). Plus rarement, ce verbe permet au contraire à un adolescent de regretter son trop jeune âge pour entreprendre une action spécifique $\left(^{42}\right)$.

L'adjectif şahrum, construit sur la même racine ŞHR, accolé au mot ahum «frère», sert à situer selon leur âge les membres d'une fratrie ; l'expression ahum şahrum est donc à traduire par «jeune frère» $\left({ }^{43}\right)$.

\section{2. Élever les enfants}

L'éducation des enfants se résume, si l'on en croit les archives des marchands de Kaniš, en leur entretien, les sources étant à peu près silencieuses sur une éventuelle formation scribale ou marchande. Les personnes chargées de l'éducation des enfants sont naturellement les parents. Mais l'éclatement des familles entre Aššur et l'Anatolie a amené parfois des situations particulières, où les jeunes enfants sont confiés à d'autres membres de la famille.

\subsection{Responsables des enfants}

\subsubsection{Père et mère}

Les parents, bien entendu, sont les premiers responsables de leurs enfants, et à ce titre, ils doivent assurer leur entretien et les éduquer. Les enfants grandissent à l'intérieur de la maison, centre du foyer ${ }^{(44)}$. Il est fort probable que certaines familles aisées embauchent une nourrice pour les assister auprès des plus jeunes enfants. Celle-ci serait alors rémunérée par une somme d'argent $\left({ }^{45}\right)$.

(39) ICK 1 32, 24-26: ta-ar-bi-tám, ú-ku-ul-ta-ša, sa-bu, «(Concernant) l'argent pour l'elever, sa nourriture, c'est

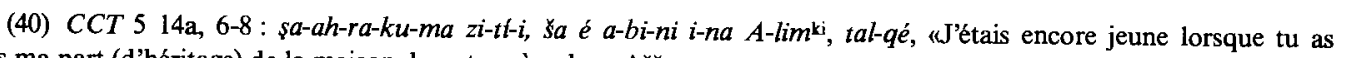
pris ma part (d'heritage) de la maison de notre pere dans A Ašurm.

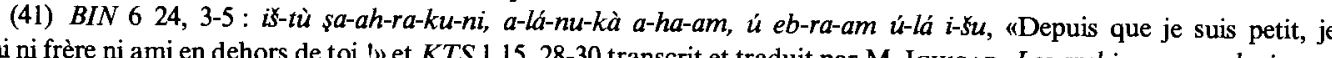
du marchand Imdżlum, Paris 1981, 327 329. 28-30 transcrit et traduit par M. ICHISAR, Les archives cappadocienn (42) Tel est le cas de Sas

(42) Tel est le cas de Săt-Assur, qui déplore le fait de ne pouvoir entreprendre une action en justice, TTC 26, 27, texte reédité dans C. MicheL, «Réédition des Trente Tablettes "Cappadociennes" de G. Contenau,, RA 80 (1986), (...)m.

et traduits par M. ICHISAR, Les archives cappadociennes (44) CCT 2 36a, 23-26, ci-dessus note 35 .

(45) TC 3 207, 24:1 1/2 GíN mu-sé-ni-iq-tum, «1 1/2 sicle (d'argent, dépenses pour) la nourrice» Selon $\mathrm{K}$ HECKER, GKT 55b, il pourrait s'agir ici d'un nom propre et non d'un nom de métier; ce document offe l'unique attestation d'une nourrice dans les tablettes paléo-assyriennes. 
Le père est le tuteur légal des enfants, et à ce titre il décide de l'avenir de ses filles ; c'est lui qui les marie ${ }^{\left({ }^{6}\right)}$. De même, Pūšsu-kēn doit présider la cérémonie au cours de laquelle sa fille doit entrer au service de la divinité $\left({ }^{47}\right)$. Cependant, les plus jeunes sont généralement aux soins de la mère et, lors d'un divorce, la femme conserve souvent la garde de ses enfants. Un texte pourtant témoignerait du cas inverse. Il s'agit de l'acte de divorce de Pilah-Ištar et de son épouse secondaire Walawala. Absent fréquemment de Kaniš, Puzur-Ištar a laissé la mère de Walawala et ses deux frères pourvoir à l'entretien de sa fille, Lamassī, et de sa femme. Au moment de la séparation, Pilah-Istar décide d'emmener sa fille Lamassī avec lui à Ašsur ; il règle donc les dépenses relatives à sa nourriture et à son éducation à la famille de sa femme ${ }^{(48)}$. Toutefois, ce cas demeure exceptionnel ; le plus souvent, la femme garde auprès d'elle ses enfants, surtout lorsqu'ils sont encore jeunes. Certains enfants sont désignés par rapport a leur mère. A partir d'un certain âge, les garçons pasent sous le contrôle du père, tandis que les filles restent auprès de leur mère jusqu’à leur départ de la maison décidé par le père.

Au début du commerce à longue distance, lors de la création des comptoirs commerciaux, les marchands assyriens de la première génération qui partent exercer leur métier en Anatolie laissent sur place épouses et enfants en bas âge pour une durée non définie. Tandis que leurs maris s'installent en Anatolie, les femmes restent donc à Aššur, élèvent leurs plus jeunes enfants et assurent leur entretien. Les soucis matériels relatifs à l'éducation des enfants occupent une et assurent leur entretien. Les soucis materiels relatifs à l'education des enfants occupent une
bonne part de la correspondance féminine. Mais, lorsque la femme se met à voyager et qu'elle rejoint son époux en Asie Mineure, elle emmène généralement avec elle ses enfants et le cercle familial est ainsi reconstitué $\left({ }^{49}\right)$.

\subsubsection{Responsabilité des grands-parents : le cas d'Aššur-idt}

Dans certains cas, où l'éclatement des familles entre Aššur et l'Anatolie centrale s'ajoute au décès de la mère la responsabilité de l'éducation des enfants du couple incombe aux plus proches parents, tels les grands-parents. C'est ainsi que les enfants d'Aššur-nādā ont été élevés par leur grand-père, Aššur-idī, fils de Šū-(i) $\overline{1}(A K T 140)$. Aššur-idī eut au moins deux fils. L'aîné, Aššrur-taklāku, est décédé relativement jeune ( $A K T$ 1 69). Le second, Ašsur-nādā, se marie successivement à Ašsur avec une Assyrienne ${ }^{\left({ }^{0}\right)}$, puis à Kaniš, avec une Anatolienne du nom de Sišahšušar. Cette dernière, considérée comme l'épouse secondaire d'Aššur-nādā met au monde deux fils et une fille ${ }^{\left({ }^{5}\right)}$. Elle habite Kanišs, et reçoit plusieurs lettres de son

(46) C. Michel, NABU 1997/40 et VS 26 64, lettre de Šü-Hubur qui demande à son collègue Pǚsu-kēn de ne pas retenir trop longtemps son futur gendre.

(47) BIN 4 9, CCT3 20, RA 59 (1965), 159.

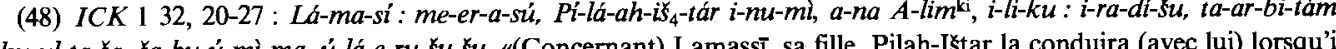

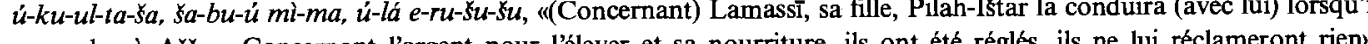

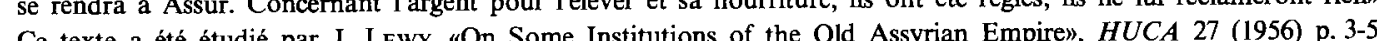

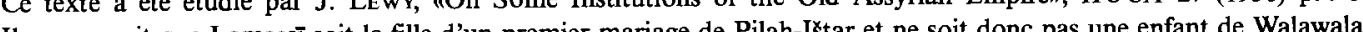

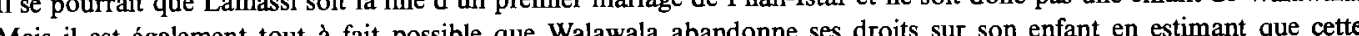
dernière aura une vie plus aisée à Ašsur. (49) P. GareLLl, «Trois tablettes cappadociennes du Musée de Rouen», RA 51 (1957) p. 6, texte HG 75, 2124: a-pu-tum a-pu-tum, iš-tí a-li-ki-im pá-nim-ma, (...) té-eb-e-ma : a-tal-ki-im, sú-ha-ra-am lá té-zi-bi-im, ue t'en prie, sil te plât mets-toi en route avec la prochaine caravane (...) et viens! Ne laisse pas le petit !).

(50) Peut-Atre s'agit-il de Sarrat-Ištar, attestée comme femme d'Ał̌̌ur-nādā dans le texte BIN 488 ?

(51) Il s'agit d'Assur-nēmedi (KTS I $8 \mathrm{~b}$ et OIP 27 56) et Anina (KTH 26 et Prague I 490). La fille, quant à elle, se nome IStar-Lamass, et elle est attestée comme l'épouse-amium de Puzur-Istar (Prague I 490). mari avant de le rejoindre dans une localité anatolienne inconnue, où il s'est finalement installé (52). L'épouse principale d'Aššrur-nādā disparaît sans doute très tôt, alors que ce dernier demeure déjà en Asie Mineure. Son fils, Iddin-Ištar, et ses filles, se trouvent orphelins à Aššur. C'est alors qu'Ašur-idī intervient et se charge de l'éducation de ses petits-enfants ( ${ }^{(53)}$ ). Il les élève donc, s'inquiète pour eux et les nourrit. Une partie de leur entretien est toutefois réglée par leur père. En effet, Ašsur-idí établit des comptes à l'intention de son fils et justifie ainsi les dépenses effectuées sur l'argent de ce dernier ${ }^{\left({ }^{54}\right)}$. Ces comptes sont d'ailleurs tenus en parallèle par des collègues d'Ašsur-nādā, vraisemblablement chargés de reverser la pension des enfants à Ašsur-idī (55).

Il est intéressant de noter que, dans le cas présent, Ašsur-idī, le grand-père, s'est complètement substitué au père des enfants, à tel point que son petit-fils, Iddin-Istar est identifié par rapport à lui et non à son père comme le veut la coutume. En effet, le texte $A K T 142 \mathrm{a}$, le présente comme «dumu» Ašsur-idī ( $\left.{ }^{56}\right)$. Quoi de plus naturel, finalement, que le petit-fils s'identifie plutôt à son grand-père, puisque c'est ce dernier qui les a élevés, lui et ses sœurs.

\subsubsection{Autres personnes responsables de l'éducation des enfants}

Beaucoup d'autres membres de la famille plus ou moins éloignés interviennent tour à tour dans l'éducation des enfants, en fonction de chaque schéma familial. A défaut de parents ou de grands-parents, les aînés prennent soin des plus jeunes, les frères de leurs sœurs, ou encore, les oncles et tantes de leurs neveux et nièces.

En l'absence du père, il est en effet fréquent que les fils de la famille décident de l'avenir de leur sœur en lui choisissant un époux ${ }^{(57}$ ), ou encore qu'ils mettent à exécution les engagements matrimoniaux pris par le passé. C'est en ce sens qu'Ahu-waqar s'adresse à Zuppa son futur beau-frère : "Ma sœur a grandi, viens ici et épouse ma sœur à Kanisl》 $\left({ }^{58}\right)$. Dan certains cas, les fils se contentent d'assister leur mère dans ces démarches et c'est avec celleci qu'ils organisent les épousailles de leur sœurs (59).

(52) CCT 3 7a, $A K T 115, T C 247, V S 26$ 19, RA 60 (1966) 140.

(53) Ce dossier a été en partie étudié par M. T. LARSEN, OACC p. 99 ; voir les textes $K U G 27, C C T 36 \mathrm{~b}$ ou $T C 393$

(54) KUG 27, 50-53: 1 ma-na kù-babbar [a]-ك̌si-im 2 gut $_{4}^{\text {háa }}$ ù ù-ti-tim, a-na me-er-e $3 a-k u-l i-i m$ ag-mu-ur, «J'ai dépensé 1 mine d'argent pour l'achat de 2 bueufs et des céréales pour nourrir les enfants (littéralement : les fils) (le reste de ton argent...) $)$.

(J5) KTS 1 9a, 4-11 et 18-21:30 ma-na urudu, é dam-gàrrim, 1 ma-na-um 10 gín, A-š̀r-be-el-a-wa-/tum $m_{8}$, ù

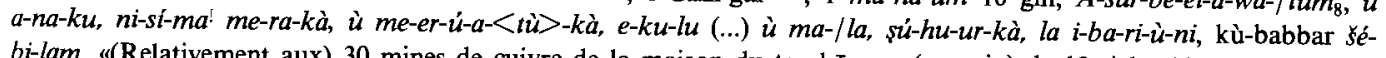
hi-lam, «(Relativement aux) 30 mines de cuivre de la maison du tamkărum, (au prix) de 10 sicles (d'argent) la mine (de cuivre), Asšurur-bêl-awâtum et moi-même avons fait (les comptes), ton fils et tes filles ont (tout) utilisé pour manger ! (56) J. (5ir) J. G. DeRko. paraitre dans $A f O$.

(57) Kt 88/K 97b, 5-6 et 11-12: a-ha-at-ni, ir-té-bi (...) a-ha-at-ni, a-na mu-tim lu ni-di-si, «Notre sœur a grand

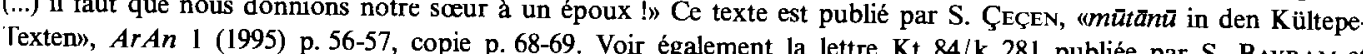
S. ÇEçEN, «6 Neue Urkunden über Heirat und Scheidung aus Kanišs, ArAn 1 (1995) publiée par S. BAYRAM el marient successivement leur trois seeurs.
(58) $\mathrm{Kt} \mathrm{i} / \mathbf{k} 120,4-7$ publié par K. BALKAN, «Betrothal of Girls during Childhood in Ancient Assyria and Anatolias,

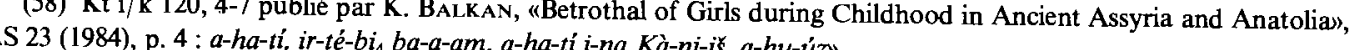
(59) TPAK $1161 \mathrm{a}+\mathrm{b}$ 
Oncles et tantes peuvent également être amenés à prendre soin des jeunes enfants de la famille. Un oncle paternel, en la personne d'Ennum-Aššur, décide ainsi de marier sa turbulente nièce et place le voile sur sa tête $\left.{ }^{60}\right)$, tandis que son épouse, Nuhšătum, s'inquiète du sort réservé à un jeune garçon et à une jeune fille, vraisemblablement ses neveu et nièce ( ${ }^{(61)}$.

Enfin, fréquemment, les collègues et représentants d'un marchand interviennent matériellement de façon ponctuelle, avançant les fonds nécessaires à l'entretien de ses enfants. On l'apprend par les notes de frais qu'ils s'empressent de transmettre au père afin d'être remboursés.

\subsection{Principales préoccupations des responsables vis-à-vis des enfants}

Tous ces adultes, en charge de l'education d'enfants, font part, dans leurs missives, de leurs préoccupations majeures quant à leurs responsabilités. Ils doivent assurer l'existence des enfants, les nourrir et les vêtir. En cas d'absence, ils s'enquièrent de leur santé et demandent qu'on les protège. Enfin, ils se soucient de leur éducation morale et désirent assurer un avenir aux enfants et plus particulièrement aux filles.

\subsubsection{Bonne santé et protection de l'enfant}

L'éclatement du noyau familial empêche souvent l'enfant de grandir au sein d'une grande famille unie. Afin de pallier cette situation, parents, grands-parents, oncles, tantes et tous les proches correspondent afin d'échanger des nouvelles des plus jeunes. Nombreuses sont les lettres paléo-assyriennes qui se terminent par la formule rassurante: "Ta maison et tes enfants se portent bien !» $\left({ }^{62}\right)$.

L'éloignement entre Ašsur et l'Asie Mineure ralentit les courriers, et certains parents s'inquiètent parfois de la raréfaction des nouvelles concernant leur famille; ils insistent pour obtenir des renseignements sur cette dernière: "Je ne sais même pas si ma jeune femme et la toute petite fille sont mortes ou vivantes! Vous (êtes) mes frères. Si elles sont vivantes (...) je pourrai donner (le montant) de leurs dépenses» ${ }^{(63)}$. En cas de longue absence, certains marchands demandent à plusieurs proches et collègues de protéger femme et enfants ${ }^{\left({ }^{64}\right)}$, de vérifier qu'ils ne manquent de rien, et éventuellement, d'avancer l'argent nécessaire à leur entretien ( ${ }^{(5)}$. Quelques-uns, enfin, ont recours à des actes d'ordre religieux pour assurer la santé et la prospérité de leur famille, et plus particulièrement de leurs enfants, par la rédaction d'une inscription votive ${ }^{(6)}$.

(60) $A K T 3$ 80, 21-25 cité par C. MıсHEL, NABU 1997/40. Les premiers exemples assyriens du port du voile par la femme mariee remontaient jusquà présent aux lois médio-assyriennes, G. CARDASCIA, Les lois assyriennes, LAPO 2, Paris 1969, \& 40, p. 201-205.

(61) $A K T 379$ et 80 et ma recension de cet ouvrage à paraitre dans $A f O$

(62) bêt-ka u şuhur-ka salim/salmu: BIN 4 91, 35, CCT 238 et VS 269 . Voir également CCT 428 b, 27 : sú-uh-ru-um sa-lim ù $n i-n u$, «Les enfants ainsi que nous-mêmes (sommes) en bonne santé», ou encore BIN 617 ,

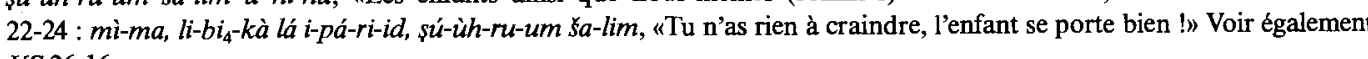
$S 2616$

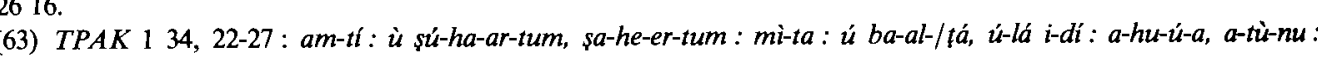
šu-ma : ba-al-tá (...) ga-mar-št-na : ta-da-na-am.

(64) ICK 1 65, 15-18: a-hu-ni a-ta, a-sé-er : a-ma-ti-ni, ú şú-uh-ri-kà, e-kà : li-li-ik, "Tu (es) notre frère, protègc nos jeunes femmes et tes enfants».

(65) TPAK 1 22, 23-28: i-séer : am-tim, ù sú-üh-ri-im, e-k[u-n]u li-li-kam, dam na-hi-da-ma, a-na gam-ri-im un tì-uš-ta-di-in, «Protegez la jeune femme et l'enfant. Surveillez la femme et tu dois lui faire donner (ce qu'il faut)

486, publíe par L. ЈАКов-Rost et H. FreYDAnK, «Eine altassyrische Votivinschrift”, AoF 8 (1981), 326 citée ci-dessus note 13

\subsubsection{Nourrir les enfants}

L'éducation des enfants concerne principalement leur entretien, et une bonne partie des lettres mentionnant l'existence d'enfant de nourir est exprimee par le factitif (III) du verbe akăum, «manger» et s'applique généralemen leur féroce appétit ( $\left.{ }^{68}\right)$. période indéterminée. Assur-idì chiffre ainsi la consommation de ses petits-enfants, sur un des rations des compte de leurs enfants change radicalement les données. Une femme se plaint en ce sens auprès de Pūšu-kēn (69): «(Si) les servantes consomment 20 litres, alors moi aussi je consomme bien 20 litres! Là où on nervantes (généralement) pas les noms des enfants, eh bien toi non plus tu na dois pas les prononce par ici !' L'auteur de cette lettre estime qu'elle ne doit pas être moins pas les prononce servantes, et que par conséquent il ne faut pas comp la distribut que par conséquent il ne faut pas comptabiliser les enfants de ces dernières dans

Argent ou céréales représentent les moyens dont doit disposer la personne responsable de l'éducation des enfants ; cette nourriture peut apparaître sous la forme de rations-iprum (70). L'absence de moyens ou le manque de nourriture constituent l'un de ses principaux sujets d'inquiétude, en témoigne le ton pathétique de certaines lettres : «Que donnerai-je aux enfants ? Que donnerai-je à notre mère ? Que dirai-je pour ma nourriture ? (...) Mes yeux sont devenus sombres (à force d'essayer de trouver) de la nourriture pour moi et pour les enfants !n (71). Afin d'apitoyer leurs correspondants sur le sort réservé aux plus jeunes du fait de leur négligence, mar inanition comme synonyme de pénegligence, enfants vont mourir de faim !» (72).

\subsubsection{Vêtir les enfants}

Outre le vivre et le couvert, les responsables d'enfants doivent leur procurer une garderobe, celle-ci se résumant sans doute à un ou deux vêtements adaptés à leur taille. Parmi les différents types de tissus recensés, il existe un túg sa şuhrim, «étoffe pour enfant»" ( ${ }^{73}$ ), qui correspond sans doute non pas à un élément de prêt-à-porter, mais plutôt à un coupon dont la taille se prête à la confection d'un vêtement d'enfant. En effet, certains textes précisent que l'étoffe doit être destinée à habiller un enfant: «une étoffe pour vêtir l'enfant» (74).

(67) C. Micher, "A table avec les marchands paléo-assyriens», CRRAI 39, Heidelberg 1997, p. 96. BIN 411 , 1819 : sú-hu-ur-kà, ú-sa-kal, «Je vais nourrir tes enfants». Voir également BIN 4 67, $12-13$ (lu tù-sa-ki-uls) ou KTS 8b, 3-9 (ú-sa-kal-su).

(68) $K T S 19$ a cité ci-dessus note 17 : «Ton fils et tes filles ont (tout) mangé».

(69) BIN 4 22, 22-25: gemé 20 silà e-kà-lá, ú a-na-ku 20 silà a-kál, a-ni su-mì sú-uh-ri-im, lả i-za-ku-ru a-na(70)-

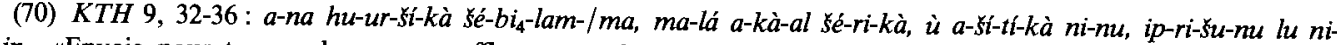
di-i $i_{5}$, «Envoie pour ton garde-manger suffisamment de nourriture pour ta femme et tes enfants, afin qu'on leur (71) $B I N$ 6 183,9-17

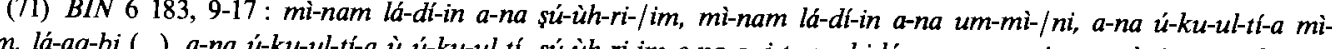

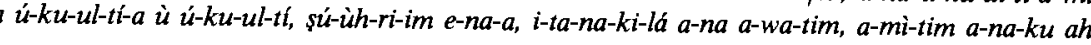

(72) BIN 6 197, 14-15: sú-ìh-ru-um i-na bu-bu-tim, i-mu-a-at-ma, ou encore BIN 6 124, 11-13.

(73) túg sa suhrim: $A K T 252,9-10$ ou BIN 6 249, 9. Voir également BIN 4 68, 10-11: 1 túg, kam-sí-um

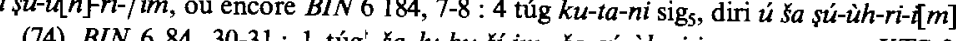

作 
des pièces de vêtements, quelques enfants de familles aisées reçoivent des éléments de parure comme des boucles d'oreilles en or ou des croissants de lune en cuivre (75).

\subsubsection{Education morale et intellectuelle}

En dehors de l'entretien matériel des plus jeunes, il convient de leur apporter une formation artisanale ou intellectuelle, de leur inculquer le sens des valeurs et de leur assurer éventuellement un avenir. Les filles fréquentent sans doute, dès leur plus jeune âge, l'atelier de tissage de leur propre demeure, où elles apprennent les rudiments du métier ${ }^{(76)}$. La formation des garçons n'apparaît presque jamais dans les textes. Le seul témoignage connu provient du fils aîné de Pūšu-kēn. Alors qu'il est resté auprès de sa mère à Aššur, il apprend à écrire et compter auprès d'un maître. Il écrit en ce sens à son père et utilise des signes et des formes verbales recherchées comme preuve de son apprentissage $\left({ }^{77}\right)$ : "Assurément, nous apprenons l'art du scribe. Envoie-moi une (étoffe)-epattum pour mon maître !». A l'appui de cette formation scribale et mathématique, quelques textes scolaires ont été retrouvés à Ašsur et à Kaniš $\left({ }^{78}\right)$, et c'est vraisemblablement en suivant leur père en Anatolie que les jeunes garçons apprennent et maîtrisent peu à peu leur futur métier de marchand.

L'éducation morale et religieuse se fait à la maison, et fréquents sont les adultes qui se plaignent de leur échec dans ce domaine face à l'irrespect de leur progéniture. Ašsur-idī, qui a tout sacrifié à l'éducation de ses petits-enfants, se plaint de leur ingratitude auprès de son fils, Aššur-nādā (79) : «J'ai élevé ton fils, mais il (m'a dit) ceci : "Tu n'es pas mon père !", il s'est levé et il est parti. J'ai également élevé tes filles, mais elles (m'ont dit) ceci : "Tu n'es pas notre père !". Trois jours plus tard, elles se sont levées et son parties chez toi, alors il faut que je connaisse ta décision !» En quittant leur grand-père, les enfants d'Ašsur-nādā auraient donc utilisé les formules de reniement classiques des ruptures de contrats d'adoption, signifiant par là qu'ils refusent désormais la tutelle du grand-père ${ }^{\left({ }^{80}\right)}$. Imdīlum, quant à lui, estime ne pas avoir obtenu le résultat souhaité avec sa fille, à laquelle il reproche son manque d'attention envers ses fils et lui-même, et avec l'un de ses fils, qui ne respecte personne et ne pense qu'à boire et à manger ${ }^{(81)}$.

(75) KTS 2 23, 13-14 citée ci-dessus note 19. BIN 6 175, 16-18: 2 ma-na hu-sa-ú: i-a-u-tum, a-na Ar-wa-tim a-na $3 /$ sú-/e-na-tim, a-na sú-uh-ri-im, «2 mines de déchets (de cuivre) m’appartenant pour Arwatum pour 3 croissants de lune pour l'enfantw. Ce texte est édité par A. UlshOFER, Die Altassyrischen Privaturkunden, FAOS B. 4, Stuttgar $1995, \mathrm{n}^{\circ} 171$.

(76) H. Limet note qu'à l'époque d'Ur III, les enfants sont très tôt mis au travail, "La condition de l'enfan en Mésopotamie autour de l'an 2000 avant J.-Ci., dans A. Théodorides, P. NASTER et J. Ries (éds.), L'enfan dans les civilisations orientales, Leuven 1980, p. 11-12.

(77) CCT 4 6e, 4-7: dub-sartän wa-di, lá-am-da-ni, e-pá-tá-am, a-na um-me-a-ni-a, šu-bi-lam

(78) V. DonsAz, «Four Old Assyrian Tablets from the City of Assuns, JCS 26 (1974), p. 81-87, v. DonBAZ, "More Old Assyrian Tablets from Ałsun», Akkadica 42 (1985), p. 123 , K. HECKER, «Schultexte von Kültepe», dan M. J. Mellink, E. Porada et T. ÖzoUc (éds.), Aspects of Art and Iconography: Anatolia and its Neighbors Studies in Honor of Nimet Özzü̧, Ankara 1993, p. 281-291, K. HECKER, "Schultexte aus Killtepe : ein Nachtrag", 1996) à paraître.

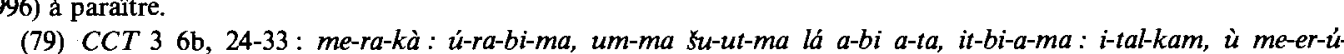

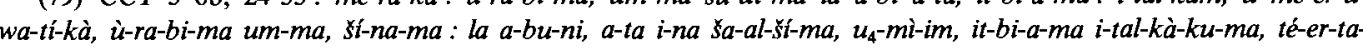
$k \grave{a} l u i-d i$, et le texte $T C 393$.

(80) $\mathrm{CH}$ \& 192, ainsi que la série ana ittišu, MSL 1 (1937), p. 23-24.

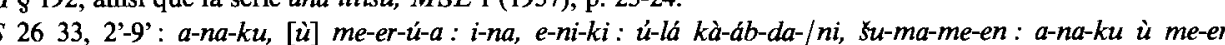
ú-a : i-na, e-ni-kà (!) : kà-abb-da-ni, ùu a-na-ku : ki-ma, me-er'-ú-a-tim ù-kà-bi-/id-ki-me-en, «Moi-même [et] mes fils,
Soucieux de l'avenir de leurs enfants, les parents promettent en mariage leurs garçons et leurs filles encore jeunes, le mariage devenant effectif lorsque la fille rejoint la demeure de l'epoux $\left({ }^{82}\right)$. Mais cette promesse peut être défaite par le jeune homme par une simple déclaration ou encore par un refus de la jeune fille $\left({ }^{83}\right)$.

Certaines filles, avant d'avoir atteint l'âge du mariage, sont vouées à une divinité et deviennent prêtresses ( ${ }^{84}$ ). Les garçons, quant à eux, s'installent progressivement en Asie Mineure où ib se marient et exercent à leur tour leurs activités commerciales.

\section{Le STATUT DE L'ENFANT}

\subsection{Enfants libres et enfants esclaves}

L'essentiel des données analysées jusqu'à présent concerne des enfants de marchands libres mais les données textuelles font également mention d'enfants d'esclaves. Le statut d'un enfant est généralement déterminé par sa filiation: un enfant d'un couple d'esclaves revêt lui-même le statut d'esclave. Toutefois, certains enfants de parents libres ont pu entrer en servitude à
la suite de l'endettement de leurs parents.

\subsubsection{Les enfants d'esclaves}

Les enfants héritent du statut de leur mère. Au début de leur vie, les petits esclaves n'apparaissent que très rarement dans les documents, car ils ne sont pris en compte que lorsque eux-mêmes constituent une force de travail et peuvent alors être séparés de leur famille. Le plus jeunes sont laissés à leur mère, qui les élève tout en travaillant. Lorsque Pūšu-kēn confie à sa femme une servante et son enfant, il précise que le petit doit être élevé dans la maison, et donc auprès de sa mère ( ${ }^{85}$ ). Mais, surtout, les rations alimentaires distribuées aux femmes comprennent parfois un supplément pour leurs plus jeunes enfants (BIN 4 22).

Dans la mesure où les enfants d'esclaves demeurent auprès de leur mère, il serait logique de les comptabiliser dans la distribution des rations alimentaires aux serviteurs. A en croire les propos de Sāt-Ašur, cela ne semble toutefois pas être le cas, sans doute en ce qui concerne les plus jeunes. En effet, la ration d'une servante, vraisemblablement mensuelle, serait inférieure

nous ne sommes pas importants à tes yeux. Si moi-même et mes fils nous étions importants à tes yeux, alors moimême, je te respecterais comme une fille !n, et TC 357 édité par M. ICHISAR, Les archives cappadociennes du marchaid (82)

(82) Kt 88/K 97b publié par S. ÇEÇEN, umūtänū in den Kültepe-Texten», ArAn 1 (1995), p. 56-57, copie p. 68

(83) $\mathbf{K t ~ i} / \mathbf{k} 120$ publié par K. BaLKan, «Betrothal of Girls during Childhood in Ancient Assyria and Anatolia»,
$A S 23$ (1984), p. 4, et BIN 6104 . (84) Voir le det $B I N 6104$

Betrothal of Girls during Child à fille de Püsu-kēn, BIN 4 9, CCT 320 et RA 59 (1965), 159. Selon K. BaLkan, l'enfant sous la protection du diew in Ancient Assyria and Anatolia», AS 23 (1984), p. 3-5, les expressions «mettre prendre la première formulation au sens propre, d'autent pluatent. Néanmoins, il n'y a aucune raison de ne pas kēn fut effectivement prêtresse. (85) $C C T 2$ 36a, 20-26: a

ki-im, a-pu-tum sú-ha-ra-am, a-na : a-nu'-na-šu: sú-ha-<ra>-am, ù am-tám [mi]]-ma a-nim, Dan-a-sùr i-ra-di-aet la servante, tout cela, Dān-Ašsur te le conduit. Je t'en prie tué-ra-bétim-ma, li-ir-bi, «En outre, ensuite, l'enfan grandir à l'intérieur de la maison». 
à 20 litres de céréales ${ }^{86}$ ). Šăt-Aššur estime être moins bien servie que les servantes, car ces dernières recevraient, contrairement à la tradition, un supplément pour leurs enfants, mettan la ration mensuelle à 20 litres ${ }^{\left({ }^{7}\right)}$.

\subsubsection{Les ventes d'enfants}

Les enfants d'esclaves interviennent principalement dans les documents relatant une vente d'esclave, lorsque, avec ou sans leur famille, ils font eux-mêmes l'objet de la transaction. Ces textes, étudiés par B. Kienast ${ }^{88}$, relèvent essentiellement de deux catégories. Les moins nombreux concernent le commerce d'esclaves, tandis que la majorité renvoie à la cession des enfants et parfois de leur famille pour une dette non éteinte. Les simples ventes d'enfants concernen souvent des filles ${ }^{89}$. Les rares prix recensés dans ces ventes varient considérablement, sans qu'il soit possible de faire un quelconque lien avec leur âge, que l'on ne sait pas estimer. Ainsi, les prix relevés por (ICK $127 \mathrm{~b}$ ), une fille et sa mère étant vendues par ailleurs pour $371 / 2$ sicles d'argent. Seule une notice personnelle comptable illustre la vente de plusieurs jeunes enfants avec leurs mères. Selon ce document, 4 femmes ont été vendues avec leurs 9 enfants, dont l'un est un jeune garçon. Cette précision indique sans doute que ce dernier a une valeur supérieure aux autres enfants. Le prix de ces 13 esclaves étant donné en nature - pierres, laine, étoffes et mouton - il est impossible d'en estimer le montant en argent (90). En marge de cette catégorie documents, le texte TC 3254 concerne la vente d'une famille d'esclaves engagée dans un prêt ( ${ }^{91}$ ).

\subsubsection{Les enfants, garantie d'une dette}

Les autres ventes d'enfants font suite au surendettement de familles ayant une vie très précaire. Un père, ou un couple, contractant une dette, se voit parfois dans l'obligation de garantir l'emprunt avec ses différentes possessions. Parmi ces dernières figurent généralement ses propriétés mobilières et immobilières, mais parfois également son épouse et ses enfants. Selon le cas, soit l'emprunt est directement contracté par l'ensemble de la famille et de la maisonnée ( ${ }^{92}$ ), de contrat $(93)$

(86) Selon P. VIILARD, ARMT XIII, p. 554, à Mari, les rations distributées aux tisseuses varient entre 3 et 10 litres de céréales par mois.

(87) $B I N 422$, cf. ci-dessus, note 69

(88) B. KIENAST, Das Altassyrische Kaufvertragsrecht, FAOS Beiheft 1, Stuttgart 1984.

(89) $\mathrm{Kt} \mathrm{a} / \mathbf{k} 554$ est cité par K. BALKAN, "Cancellation of Debts in Cappadocian Tablets from Kültepe», dans Anatolian Studies Presented to Hans Gustav Gütterbock on the Occasion of his 65th Birthday $=$ Studies Gütterbock, Istanbul 1974, p. 30, note 13, et réédité par B. KIENAST, FAOS Beiheft 1, n. 4, p. 108-109. Il s'agit de l'achat d'une suhärtum; son prix n'est pas mentionne.

(90) TC 3 183, 1-7: 65 áb-ni, sa-áp-tum 31 túg, 80 è-me-ru, 3́t-im 4 a-ma-tim, ú 9 șí-ìh -ri-im, «65 pierres, de la laine, 31 etoffes, 80 moutons: prix de 4 sentes et 9 a

(91) TC 3 254, 6-17 étudié par B. Kienast, FAOS Beiheft 1, n. 37.

(92) TC 3 238, 1-9: 3 ma-na kù-babbar, li-tí : sigg, i-şé-er : A-ta-pì-lá, $A-<l a>-w a-a ̈ s-h i$ a-sit-ti-su, me-er-e-su:

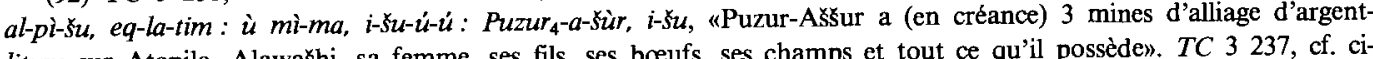
litum sur Atapila, Alawashi, sa fem

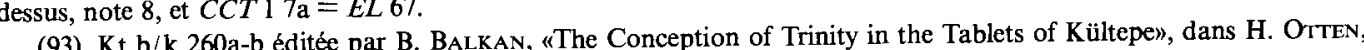
(93) $\mathrm{Kt} \mathrm{b} / \mathrm{k} 260 \mathrm{a}-\mathrm{b}$ éditée par B. BAIKAN, «The Conception of Trinity in the Tabers of Kustern Studies in Honour of E. AKURGAL, H. ERTEM et A. SUEL (eds.), Hittite and other Áá Sedat Alp, Ankara
Un individu qui engage sa femme et ses enfants pour garantir une dette contracté, met à la disposition de son créancier jusqu'au remboursement de l'emprunt (94). Dans le cas où il ne peut éteindre sa dette, les personnes engagées deviennent propriété du banquier et perdent leur liberté. Tel est le cas d'une famille agestien et fille, qui n'ayant pu régler une dette, doit servir le créancier ( ${ }^{95}$. Selon couple et de sa remour n'ayant pu ce texte, seul le certaines mesures royales comme les la famille de l'esclave et lui rendre sa liberté. Toutefois, assyriennes $\left({ }^{96}\right)$ pouvaient rendre la liberté àssions de dettes attestées par les archives paléo-

\subsection{Adoption et héritage}

Les archives privées des marchands de Kaniš documentent plusieurs sortes de filiation. ôté des enfants légitimes du couple, certains enfants ou epouse secondaire, d'autres arrivent dans la famille par le biais de l'adoption. Senton cas, les droits de succession de ces différents enfants vaile par

\subsubsection{Enfants légitimes et enfants de servantes}

Selon les lois médio-assyriennes, de quelques siècles postérieures $\left({ }^{98}\right)$, le mariage es de l’épouse. de lepouse. Néanmoins, lhomme peut avoir une épouse secondaire afin de s'assurer unes descendance. La tradition paléo-assyrienne tient compte de la situation ás de s'assurer une u marchand par e marchand épount vers la bigamie ou amtum en Anatolie (99). un ou plusieurs héritiers. L'éventuelle stérilité mariage, l'époux espère généralement obtenir d'un contrat de rente envisagée par la rédaction elle ne lui a pas procen épouse-ǎšătum Hatala. Si dans deux ans, lui a lu a pas proche servante et plus tard, ansè lui avoir procure un enfant par cet homme, elle pourra la vendre la où elle plus tard, après Dans le cas présent, l'épouse principale n'ayant pu donner naissance à un le voudray ( ${ }^{100}$ ). une esclave génitrice qui, après avoir eu un enfant de son mari, reste à la disposition de la

(94) $K T K 95$ et 96, ci-dessus note 9, ainsi que le texte $C C T 110 \mathrm{~b}+11 \mathrm{a}$ ( $=E L$ 15), 15 18: Ku-uk-ra-an,

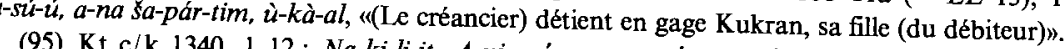

(95) Kt c/k 1340, 1 12: Na-ki-li-it, A-ni-a ú me-er-a-sú, a-na é Us-ha-li-a, e-ru-bu, ú-ba-li-sú-nu, su-ma ra-ma-

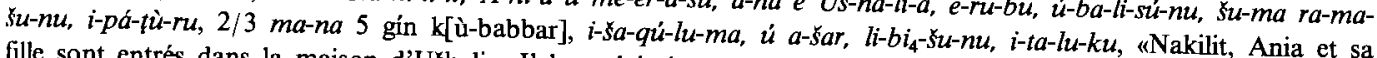
fille sont entrés dans la maison d'UShalia. Il les a laissés en vie. Si eux-mêmes (veulent) se libérer (de leur dette), ils payeront $2 / 3$ mine 5 sicles d [argent], et ils seront libres d'aller là où ils le désirenty, et les autres exemples donnés Gastov Güterbock oncellation of Debts in Cappadocian Tablets from Kultepe», Anatolian Studies Presented to Hans (96) K. Bast on he Occaston of his 65th Birthday, Istanbul 1974, p. 30-31, notes 13 et 14.

Hans Gustov Güterbock on the Occasion of his 65th Birthan Tablets from Kültepen, Anatolian Studies Presented Hans Gustav Güterbock on the Occasion of his 65th Birthday, Istanbul 1974, p. 29-41.

Ic $\$ 117$ du Code de Hammurabies à nos sources, limitent dans le temps la durée de servitude, voir par exemple

(98) G. CARDASCIA, Les lois assyriennes, LAPO 2, Paris 1969, A§ 46.

Müzesi, 1995 yillığ Ankara, p. 295-302. P. GARELLl, «New Old Assyrian Marriage Contracts», Anadolu Medeniyetleri

(100) ICK 13 analysé par H. Hirsch, „Eine Kleinigkeit Heiratsurkunde», OrNS 35 (1966), p. 279-280. 
femme stérile qui peut la revendre. Les enfants de l'esclave deviennent alors les enfants légitimes du couple, leur mère naturelle ne gardant aucun pouvoir sur eux.

En revanche, lorsque le contrat concerne une union entre un marchand et une épouse En revanche, lorsque le contrat con d'infertilité, l'achat et le choix de l'esclave génitrice secondaire amtum, il semble qu'en cas d'infertilité, l'achat et le choix de l'esclave génitrice
incombent au mari (101). Ces deux cas ressemblent en quelque sorte à une adoption des enfants naturels du père par son épouse.

Qu'en est-il du statut des enfants qu'un homme obtient par son second mariage avec une épouse-amtum? Ces derniers devraient également pouvoir acceder à l'heritage paternel. Néanmoins, il semble que les statuts de ces enfants diffèrent de ceux des enfants issus du Nén cas de Sat-Ashr, fille de Kunanila et d'AYhur-mutabbil, fils de Tel pourrait être le cas de kên, qui se plaint de ne pas hériter de son père, à moins qu'il ne soit question, dans ce cas précis, d'une limite d'âge à respecter ${ }^{\left({ }^{103}\right)}$. Dans ces différents exemples, il n'est toutefois pas véritablement question d'adoption dans la mesure où les enfants demeurent chez leur père.

\subsubsection{Adoption}

Les tablettes paléo-assyriennes offrent très peu de contrats d'adoption ; ceux-ci on été analysés par G. Eisser et J. Lewy (104). L'enfant adopté est introduit dans la famille et doit vivre et par $\mathrm{G}$. Eiser et $\mathrm{J}$. Lew $(1 / 3)$ L'enf grandir en son sein ( $A A A$ 1/38 $=E L 7)$. L'adoption ne se trs $1240(=E L$ présente ainsi le cas d'un couple d'Anatoliens qui adopte un garçon, sans doute déjà grand et exerçant une activité professionnelle. En effet, il est stipulé que tout profit que l'adopté pourra obtenir doit être rapporté au foyer sous peine d'amende $\left({ }^{105}\right)$. En outre, en cas d'endettement, le père adoptif est autorisé à vendre son fils adopté. La compensation de ce dernier intervient en revanche dans ses droits successoraux : il est l'unique héritier.

\subsubsection{Héritage}

Face à l'héritage, filles ou garçons, enfants légitimes, enfants d'épouse secondaire ou enfants adoptés, n'ont pas les mêmes droits. Le vocabulaire en matière de droits successoraux a été adoptes nent analysé par F. R. Kraus, et il n'est pas question ici d'en reprendre l'etude détaillée (106). Les termes aplum et aplūtum sont très rares en paléo-assyrien, et ils interviennent non pas dans leur contexte juridique, mais dans des lettres privées ou des déclarations $\left({ }^{107}\right)$. $(101)$ p. 6-8.

Prague I 490 édité par J. LewY, «On Some Institutions of the Old Assyrian Empire», HUCA 27 (1956) (102) Selon les cades de lois, tel est le cas des enfants d'une amtum et d'un homme marié par ailleurs, les Lois et 173 .

(103) Cf. ci-dessous et TTC 26 réedité par C. MichEL, «Réédition des trente tablettes "cappadociennes" de G. (103) Cf. ci-dessous et $T T C 26$ redite par C. M. Micher, «Les malheurs de Kunnañya, femme de marchand", Mémorial E. Bilgiç, Ankara (sous presse).

G. Eisser et J. Lewy, Die Altassyrische Rechssurkunden vom Kültepe, $M V A G 33(1930)=E L \mathbf{n}^{\circ} 7$ et 8 . (105) $T C L 1240=E L$ 8. Pour la lecture des lignes 3-6, cf. K. HECKER, GKT 138b, il faut lire le début de la ligne 4 ainsi : $k i-i_{5} l_{5}$-pá-am, uprofits.

(106) F. R. KRAUS, «Von altmesopotamischem Erbrecht», et «Erbrechtliche Terminologie im altem Mesopotamien», $S D$ IX 1969, p. 1-17 et p. 18-57.

(107) CCT 5 1ld, 18: áp-lum, ou encore VS 26 52, 7-8: NP a-na ap-lu-tim, ù-la al-qe-su, «Je n’ai pas pris NP pour héritiens.
Les enfants légitimes héritent tout naturellement des biens de leurs parents, actif et passif. Selon les rares testaments retrouvés, il semble que les filles obtiennent parfois également une part, au même titre que leurs frères $\left({ }^{108}\right)$. En d'autres occasions, la dot qui leur est attribuée constitue leur part, et, en cas de décès prématuré du père, ce sont les fils de la famille qui gèrent le mariage de leurs sœurs $\left({ }^{109}\right)$. Les enfants des épouses secondaires pourraient également hériter bien que leur statut soit inférieur à celui des enfants d'épouses principales. En face de ces derniers, il ne leur est pas toujours facile de s'imposer lors du partage du patrimoine, d'autant que, demeurant en Anatolie, ils se retrouvent loin des capitaux du patrimoine, rapatriés à Ǎšur (110). Quant aux enfants adoptés, ils ne peuvent être écartés de lo souvent par la présence d'enfants légitimes. Un paragraphe du code de Hammurabi, postérieur à nos sources, explique qu'un couple donnant naissance à ses propres enfants après en avoir adopté un autre, ne peut exclure l'enfant adopté de la succession sur les biens mobiliers ; cette obligation n'aurait en revanche pas lieu dans le cas de propriétés immobilières (111). Le contrat d'adoption $T C L 1240$ donnerait encore davantage de prérogatives à l'enfant adopté : ce dernior, dans l'hypothèse où son père adoptif $a$ un fils, hérite quand même de l'enseme : ce dernier, dans mais dans le cas présent, il s'agit clairement d'une adoption économique (112).

\subsection{De l'enfance à l'âge adulte}

A différents degrés, tous les enfants peuvent donc revendiquer une part du patrimoine familial. Néanmoins, certains d'entre eux ne peuvent en jouir immédiatement, n'ayant pas atteint l'âge requis pour hériter. Un procès-verbal établi entre deux frères indique que l'aîné perçoit la part d'héritage du benjamin afin de la gérer tant que ce dernier n'a pas atteint la capacité légale : "Puzur-Ana (a déclaré) ceci contre Aššur-taklāku: "Tu (es) mon père, tu (es) mon
maître. J'étais encore jeune et tu as pris ma part (d'héritage) de la maison de notre père dans Aššr" (113). Aš̌ur-tjeūa et tu as pris ma part (d'heritage) de la maison de notre père dans un versement de 12 minesu reconnaît avoir perçu la part de son cadet et il règle l'affaire par un versement de 12 mines d'argent à Puzur-Ana.

Selon J. Klíma, la capacité juridique, à Mari, équivaut à l'âge d'apparition de la barbe chez le garçon $\left({ }^{114}\right)$, toutefois, ses arguments fondés sur la correspondance entre un souverain et son fils ne sont pas recevables. Les codes de lois, pour leur part ne font jamais mention d'un âge où l'enfant, de mineur, deviendrait majeur et indépendant d'un point de vue juridique.
Le code de Hammurabi effectue une distinction entre un enfant en âge d'accomplir le service

(108) Voir le testament $I C K 112$ étudié par W. von SoDEN, «Ein altassyrisches Testament», WO 8 (1976), p. 211 D. , «Assyrische Testamente», $Z A 66$ (1976), p. 202-203, RA 60 (1966), p. 131-138 repris par C. WilcKE, $Z A 66$ (1976) p. 204-208.

(109) Kt 84/k 281 publiée par S. BAYRAM et S. ÇEÇEN, «6 Neue Urkunden uber Heirat und Scheidung aus (110), $\operatorname{ArAn} 1$ (1995), p. 5

(111) C. MiCHEL, «Règlement des comptes du défunt Hurasănumu», RA 88 (1994) p. 121-128.

(111) A. Finet, Le code de Hammurapi, LAPO 6, Paris 1983, p. 108-109.

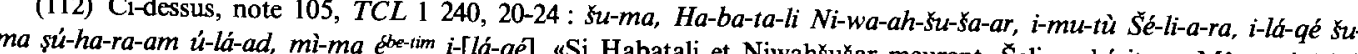
donne naissance à un garçon, il (l’adopté) recevra (quatali et Niwahsusuar meurent, Šeliara héritera Même si (elle) ainsi : e-sú-mu, ma-sú-nu sa 3 su-nu-tí, «Tout ce qu'js possedent (toute la proprietém. Les lignes 10-11 sont à lire

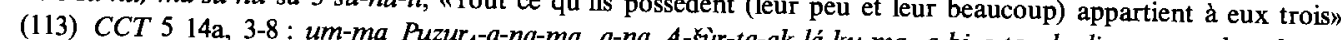
zi-tí, sa É a-bi-ni i-na A-limki, tal-qé. (114) J. K IMA, uLa position tcon

première moitié du II' millénaire avant notre ères, Aror juridique de l'enfant d'après les sources cunéiformes de Mari, 42 (1974) p. 235. L'auteur fonde sa théorie sur la correspondance 
de son père et un enfant trop jeune pour l'effectuer, et sous la tutelle de sa mère, mais le texte ne précise pas l'âge limite entre l'enfance et l'âge adulte $(\mathrm{CH} \S 28$ et 29). Les lois assyriennes, quant à elles, mettraient l’âge de capacité légale du garçon à 10 ans (A \& 43) (115).

Les lettres des marchands de Kaniš confirment l'existence d'un âge auquel l'enfant entre dans l'âge adulte et acquiert son indépendance légale, mais elles n'apportent aucun indice sur l'âge auquel s'effectue cette transition (116).

En définitive, les archives des marchands de Kaniš ne laissent qu'une petite place aux enfants, pourtant nombreux dans les familles. Les différentes études prosopographiques publiées indiquent, par couple, une moyenne de 4 à 5 garçons ayant atteint l'âge adulte. Les termes asexués utilisés pour désigner les enfants renvoient généralement aux plus jeunes, tandis que les mots distinguant filles et garçons se rapportent aux plus âgés des enfants. Ne pas précise le sexe des enfants en bas âge est une constante de la documentation mésopotamienne; une distinction entre filles et garçons est nécessaire, ces derniers ayant un rôle plus important à jouer.

Les archives privées des marchands, naturellement orientées vers les gains et les dépenses, documentent abondamment les problèmes liés à l'entretien des enfants et renforcent ainsi la prépondérance des soucis matériels causés par les enfants. Cependant, une bonne part du temp par les adultes aux plus jeunes devait se concentrer sur leur éducation, absente des consacré par les sources écrites.

Tous les devoirs des adultes envers les enfants recensés dans cette étude devaient être compensés une fois l'enfant devenu adulte, par de nombreux aspects qui n'ont pas été envisagés dans la présente étude, comme le respect dû aux anciens, l'entretien des plus âgés ou encore la pratique du culte funéraire.

Cécile Michel (EP 1730 du CNRS)

(115) M. Goneluf, La condition juridique de l'enfant en droit suméro-babylonien, Thèse de Doctorat inédite de Paris-II sous la direction de G. Cardascia, 1971, p. 304.

(116) Le texte KTS 115 edité par M. ICHISAR, Les archives cappadociennes du marchand Imditum, Paris 1981 p. 327-329, pourrait faire allusion à ce passage. voir également TTC 26 réddité par C. MicheL, «Rédition des Trente Tablettes "Cappadociennes" de G. Contenau,, RA 80 (1986) p. 127-128.
Les enfants des familles déportées de Mésopotamie du nord à Mari en ZL 11,

Résumé. - A la fin du règne de Zimrî-Lîm, au cours des années ZL 11' et 12' (1763 et 1762 av. J.-C.), deux campagnes du roi de Mari dans le nord de la Mésopotamie lui permirent de déporter population de six villes conquises. Ȧ cette occasion les scribes dresserrent des listes pour inventorier butin humain, dans lesquelles les individus étaient nommés, groupés par famille et comptés. renseignements donnés par les listes de l'année ZL 11' sont les plus nombreux et les plus précis en ce qui concerne les enfants, de sorte qu'il est possible d'estimer leur nombre et leur proportion parmi les déportés et de distinguer diverses classes d'âge, des nourrissons aux adultes.

Abstract. - At the end of Zimri-Lîm's reign, during the years ZL 11' and ZL 12' (1763 and 1762 B.C.), two campaigns in Northern Mesopotamia allowed the king to deport the population of six towns Long tablets listing the human booty were therefore written down by official scribes; these tablets give they offer of the deportes and present them by families. This study deals with the ZL 11' lists since among all the deportes and to perceive diferd is the children mong all the deportees and to perceive different age groups, from babies to adults.

À la fin du règne de Zimrî-Lîm, au cours des années ZL 11' et 12' (1763 et 1762 av. J.-C.), deux campagnes du roi de Mari dans le nord de la Mésopotamie lui permirent de déporter la population de plusieurs villes conquises. À cette occasion, les scribes tinrent des comptes précis des personnes déplacées $\left({ }^{1}\right)$. Le corpus se compose d'une trentaine de tablettes, parmi lesquelles figurent de longues listes de déportés, nommés et groupés par familles. De tel documents offrent un grand intérêt onomastique, en particulier parce qu'ils contiennent une
multitude de noms hourrites : G. Wilhelm et $T$. Richter étudient actuellement ces aspects multitude de noms hourrites: G. Wilhelm et T. Richter étudient actuellement ces aspects linguistiques. Ces textes fournissent aussi des renseignements précieux sur la composition des familles déplacées.

Les travaux consacrés aux familles de Mésopotamie se fondent en général sur l'analyse des archives privées et portent de ce fait sur des familles d'un niveau social élevé, ayant laissé des lettres ou des contrats. Rien de tel ici : même si un tableau montre que certaines familles possédaient du bétail et n'étaient pas particulièrement pauvres, ces populations, lorsqu'elles arrivent à Mari, sont réduites au pas particulièrement pauvres, ces populations, lorsqu'elles arrivent à Mari, sont réduites au rang de prisonniers dont le vainqueur dispose à sa guise. Le seul texte comparable à ceux du présent dossier est une grande liste d'époque médioassyrienne datant du $\mathrm{XIII}^{\mathrm{e}}$ siècle av. J.-C., retrouvée à Kār-Tukultī-Ninurta, étudiée par $\mathbf{H}_{\text {. }}$ Freydank $\left(^{2}\right)$; cette tablette est d'autant plus intéressante pour le sujet abordé ici qu'elle concerne aussi, mais cinq siècles plus tard, des populations hourrites.

(1) Jean-Marie Durand a confié l'étude de ce dossier à G. Wilhelm, T. Richter et moi-même, dans le cadre d'un programme Procope, et je l'en remercie vivement.

(2) Il s'agit de la liste $V S 216=$ VAT 18087+, que H. FreYDANK a commentée dans «Zur Lage der deportierten Hurriter in Assyrienn, AoF 7 (1980), p. 89-117. Les données de cet article seront abondamment utilióes cirdessous. 